\title{
Alveolar soft part sarcoma of the retroperitoneum: A case report
}

\author{
Satyam $^{1 *}$, Alpana Manchanda ${ }^{1}$, Ramya $^{1}$, Anju Garg ${ }^{1}$, Kaushik Majumdar ${ }^{2}$ \\ 1. Department of Radiology, Maulana Azad Medical College and Lok Nayak Hospital, Jawahar Lal Nehru Marg, New Delhi-110002, India \\ 2. Department of Pathology, G. B. Pant Institute of Postgraduate Medical Education and Research (GIPMER), Jawahar Lal Nehru Marg, \\ New Delhi-110002, India \\ * Correspondence: Satyam, Department of Radiology, Maulana Azad Medical College and associated Lok Nayak Hospital, Jawahar Lal \\ Nehru Marg, New Delhi-110002, India

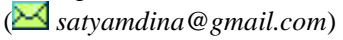

Radiology Case. 2021 Oct; 15(10):20-26 :: $\quad$ DOI: 10.3941/jrcr.v15i10.4003

\begin{abstract}
Alveolar soft part sarcoma is a rare soft tissue tumor with uncertain histogenesis. It is a slow growing tumor with a high rate of metastasis. The tumor is not easily identified as clinical symptoms are not pronounced. The retroperitoneum is a rare location of tumor, with a few cases published in literature. Surgical excision is the mainstay of treatment. Here we describe a rare case of a large retroperitoneal Alveolar soft part sarcoma in a young female with radiological and histopathological findings.
\end{abstract}

\section{CASE REPORT}

\section{CASE REPORT}

A 16-year-old female patient presented with pain in the left hypochondrium. On examination, the patient was stable. She was afebrile [temperature 37.5 Celsius] and vitals were stable [heart rate $79 \mathrm{bpm}$ blood pressure 120/80 $\mathrm{mm} \mathrm{Hg}$ record in right arm supine].

On physical examination the abdomen was soft, nontender. All blood parameters including serum alpha fetoprotein levels were within normal limits.

Computed tomography (CT) of the abdomen revealed a large well-defined solid-cystic mass of size $(9 \times 9.1 \times 8.7 \mathrm{~cm})$ in the left anterior pararenal space with multiple irregular septae within. A few linear and irregular dense calcifications were seen within and along the wall of the mass (Fig. 1A). After contrast administration, enhancement of peripheral capsule and the solid component of the tumor was seen (Fig. 1B). The perilesional fat planes with adjacent organs were preserved.
Solid visceral organs were normal on imaging. No significant lymph nodes were present.

Magnetic resonance imaging (MRI) showed a heterogeneously hyperintense mass on both T1W \& T2W sequences (Fig. 2A \& 2B) and enhancement on post gadolinium scans (Fig. 2C). There was no wash out of the contrast on the delayed phase of dynamic scans. The solid component of the lesion showed restricted diffusion on diffusion weighted imaging (DWI) (Fig. 2D \& 2E). On chemical shift imaging, there was no signal drop in the lesion.

Based upon the above mentioned CT and MRI findings, initially the possibility of retroperitoneal teratoma with malignant transformation was considered.

The patient underwent exploratory laparotomy for excision of the tumor. Intraoperative findings revealed a large, encapsulated, well vascularised tumor of solid-cystic consistency located anterior to the left kidney and posterior to 
the stomach which contained hemorrhagic fluid. Dense adhesions were found between the neoplasm and adjacent structures such as renal vessels, splenic vessels and left adrenal gland.

The tumor was a globular, well encapsulated, grey white solid cystic, soft tissue lesion measuring approximately $10 \times 9 \times 6.5 \mathrm{~cm}$ in size on gross pathological examination. Cut surface showed a well encapsulated grey white cystic friable tumor, with a wall thickness of $0.2-0.4 \mathrm{~cm}$. The normal left adrenal gland was compressed at one pole by the tumor. Calcified areas were seen within the tumor substance. Microscopic examination revealed a tumor with cells arranged in alveolar pattern separated by thin fibrovascular septae, and focally in solid sheets. Interspersed areas of calcification, hemorrhage and necrosis were identified. Individual tumor cells were round to polygonal with pale granular eosinophilic, friable cytoplasm, coarse chromatin and prominent nucleoli. Presence of intracytoplasmic crystals were noted in few of the tumor cells which were positive for Periodic Acid Schiff (PAS) with diastase. On immunohistochemistry, the tumor cells were focally positive for vimentin and epithelial membrane antigen (EMA), synaptophysin, S100, while negative for cytokeratin, epithelial chromogranin, CD10 and HMB45 (Fig. 3A \& 3B). Based on characteristic histopathological and immunohistochemical findings, final diagnosis of alveolar soft part sarcoma (ASPS) of the retroperitoneum was given.

Post-operative recovery was uneventful.

After 3 months, follow up abdominal contrast enhanced computed tomography (CECT) revealed no residual or recurrent lesion.

\section{DISCUSSION}

\section{Etiology \& Demographics:}

In 2002 WHO soft tissue tumor classification, Alveolar soft part sarcoma (ASPS) was included in the category of "Tumor of uncertain differentiation" [1]. ASPS is a rare but discrete soft tissue tumour accounting for $0.5-1 \%$ of softtissue sarcomas [2]. It is a slow-growing malignant neoplasm with unknown histogenesis. The most common location of this tumor is in the lower extremities, usually in the thigh and buttocks of a young adult. It may also occur in rare sites such as the head and neck, mediastinum, retroperitoneum, breast and orbit [3]. In children, it usually occurs in the head and neck region. The tumor affects mainly younger patients with female preponderance. The peak age of incidence of the tumor is between 15 and 35 years, but the tumor can occur in children as young as 2 years [4]

Christopherson et al first described ASPS in 1952 [5]. ASPS is also known by a variety of names, such as malignant myoblastoma, granular cell myoblastoma, and malignant granular cell myoblastoma. The origin of ASPS is considered to be myogenic with a pseudo alveolar pattern formed by aggregates of large granular cells surrounded by vascular channels. It resembles the pattern of the respiratory alveoli.

\section{Clinical \& Imaging Findings:}

The clinical symptoms are not pronounced due to slow growth of the tumor. ASPS has a high rate of early metastasis due to the high vascularity of the tumor and its microvascular invasion. It has poor prognosis in adult patients. A preoperative diagnosis of ASPS from other soft tissue tumors is important for optimal patient management and improving prognosis. If resected early, local recurrence is rare and long term survival is achievable.

Diagnostic imaging plays a crucial role in both identification and preliminary lesion characterisation, even though imaging findings are nonspecific. A definitive diagnosis of ASPS relies on histopathology, immunohistochemistry, and molecular biology. Non-contrast computed tomography (NCCT) shows iso or hypodense mass compared to the surrounding skeletal muscles with well defined margins. ASPS shows strong enhancement with central necrosis after contrast administration, in up to $75 \%$ of the cases.

In magnetic resonance imaging (MRI), the tumor is well circumscribed which can be attributed to the fibrous pseudocapsule surrounding it. The signal intensity is heterogeneous in most tumors. The tumor typically has intermediate signal intensity - mildly higher than that of muscle-on T1-weighted images and heterogeneous high signal intensity on T2-weighted images, with multiple flow voids within and around it. The intermediate or mildly high T1 signal intensity is perhaps due to its rich vascularity and intratumoral bleeding, in addition to the slow flowing blood within the tumor [1]. There is a strong and uniform enhancement with IV contrast medium. Small areas that fail to enhance may represent tumour necrosis.

Conventional angiography typically shows hypervascularity of large soft tissue mass with enlarged feeding arteries and prominent draining veins, prolonged capillary staining and delayed washout of contrast medium.

Ultrasonography (USG) reveals a variable echo pattern with large tumor vessels and poorly defined margins, which on Color Doppler Ultrasound (CDUS) shows prominent vascularity within the tumor.

CDUS, contrast enhanced computed tomography (CECT), contrast enhanced magnetic resonance imaging (CEMRI), and angiography all show increased perilesional and intralesional vascularity [4]. The presence of a large soft-tissue mass associated with large peritumoral vessels is strongly suggestive of ASPS.

The diagnosis of ASPS is confirmed by histological analysis, where it is characteristically composed of uniform organoid nests of polygonal cells with focal pseudo alveolar arrangement. The tumor cells are rich in cytoplasm, containing Periodic Acid Schiff (PAS) and diastase positive granules and typical crystals. The nuclei are round to oval, with an irregular chromatin pattern, prominent nucleoli and infrequent mitoses. ASPS is immunohistochemically negative for neuroendocrine and epithelial markers, and may be occasionally positive for myogenic markers [6]. 


\section{Treatment \& Prognosis:}

Complete surgical resection is the mainstay of therapy. Postoperative adjuvant radiotherapy and chemotherapy have been thought to be effective in reducing the risk of local recurrences and distant metastases.

\section{Differential Diagnosis:}

Alveolar soft part sarcoma needs to be differentiated from other entities:

Arteriovenous malformation (AVM): It is a vascular malformation showing large vascular components with scanty solid components and frequently fibrofatty tissue. A typical arteriovenous malformation shows rapid draining of contrast agent due to high-flow shunting and no soft tissue components. Purely venous malformations are visualized at venous angiography and not at arteriography. Haemangioma has exclusively vascular components with scanty solid components and frequently fibrofatty tissue.

Renal Cell Carcinoma (RCC): It is usually seen in adult patients above 40 years. It has a large size, heterogeneous appearance and hyper vascular nature. It has areas of necrosis and calcification. It usually shows an avid enhancement in the arterial phase with progressive washout from corticomedullary to nephrographic and delayed phase.

Adrenal Cortical Carcinoma: The patient will exhibit cushing-like symptoms. It is usually large in size and irregular shaped with a central area of necrosis and haemorrhage. It shows variable enhancement with relative retention of contrast (washout < 40\%) on delayed contrast enhanced computed tomography.

\section{TEACHING POINT}

Alveolar soft part sarcoma is a rare type of neoplasm which commonly involves skeletal muscle and soft tissue and is rarely seen in the retroperitoneum. These tumors have poor prognosis. It affects young adults and children with imaging features of a soft tissue enhancing mass with intra and peritumoral vessels. Final diagnosis is by histopathology.

\section{REFERENCES}

1. Kumar GK, Nemade H, Krishnamohan, Fonseca D, Rao LMCS, Rao TS. Rare Case Report of Alveolar Soft Part Sarcoma of the Orbit. Indian Journal of Surgical 24 Oncology. 2016;8(2):234- 239. PMID: 2854672

2. Itani M, Shabb NS, Haidar R, Khoury NJ. AIRP Best Cases in Radiologic-Pathologic Correlation: Alveolar Soft-Part Sarcoma. Radiographics 2013;33(2):5 85- 21 593. PMID: 23479715

3. Portera CA, Ho Viet, Patel S R et al. Alveolar soft part sarcoma: Clinical course and patterns of metastasis in 70 patients treated at a single institution. Cancer 2001;91(3): 9585-591. PMID: 11169942

4. Xin F Y, Rana N, Ming Z, Lang Y B. Alveolar soft part sarcoma of the retroperitoneum. J Cancer Res Ther 2010;6(1):117-119. PMID: 20479565

5. Christopherson WM, Foote FW Jr, Stewart FW. Alveolar soft-part sarcomas; structurally characteristic tumors of uncertain histogenesis. Cancer 1952;5(1):100- 4 111. PMID: 148869026.

6. Rekhi B, Ingle A, Agarwal M, Puri A, Laskar S, Jambhekar NA. Alveolar soft part sarcoma revisited': Clinicopathological review of 47 cases from a tertiary cancer referral centre, including immunohistochemical expression of TFE3 in 22 cases and 21 other tumours. Pathology 2012;44(1):11-7. PMID: 22173238

7. Temple HT, Scully SP, O'Keefe RJ et al. Clinical presentation of alveolar soft part 6 sarcoma. Clin. Orthop. 1994; 300: 213-8. PMID: 8131338

8. Kayton ML, Meyers P, Wexler LH, Gerald WL, LaQuaglia MP. Clinical presentation,treatment,and outcome of alveolar soft part sarcoma in children, adolescents, and young adults. J Pediatr Surg 2006;41(1):187-93. PMID: 16410131. 


\section{FIGURES}
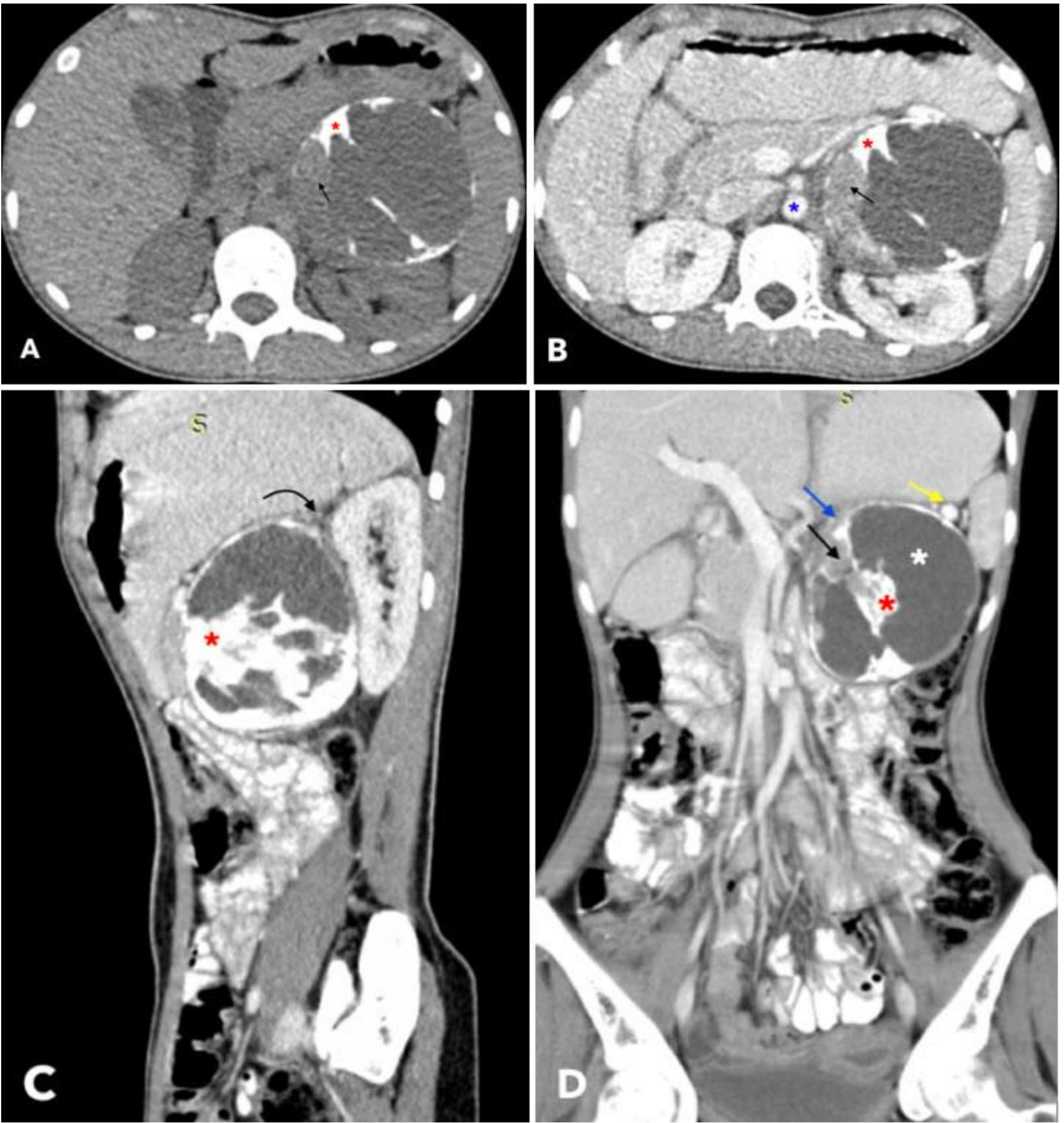

Figure 1: 16 year old female with retroperitoneal alveolar soft part sarcoma.

FINDINGS: Axial (1A) noncontrast CT images reveal a well defined rounded solid-cystic mass in the left pararenal space showing small solid component (black arrow) and coarse calcification (red asterisk) within and along the walls.

Axial (1B), Sagittal (1C), and Coronal (1D) contrast CT images show well defined rounded solid-cystic mass in the left pararenal space showing enhancing small solid component (black arrow) and coarse calcification (red asterisk) within and along the walls. Few peritumoral vessels (blue and black curved arrow) are seen. The mass is causing indentation in the left kidney.

\section{TECHNIQUE: 128 slice CT, SIEMENS SOMATOM Definition AS+}

1A: Axial non contrast CT : $166 \mathrm{~mA}, 120 \mathrm{Kv}$, slice thickness- 5mm, Window level:40 and Window width: 340

1B-D: Axial contrast enhanced CT of the abdomen in venous phase with sagittal and coronal reformat,166 mA, $120 \mathrm{Kv}$, slice thickness-5mm, Window level:40 and Window width: 340 acquired after intravenous contrast injection (80 ml of nonionic contrast Omnipaque 350mg/ml, GE Healthcare, ) USA 

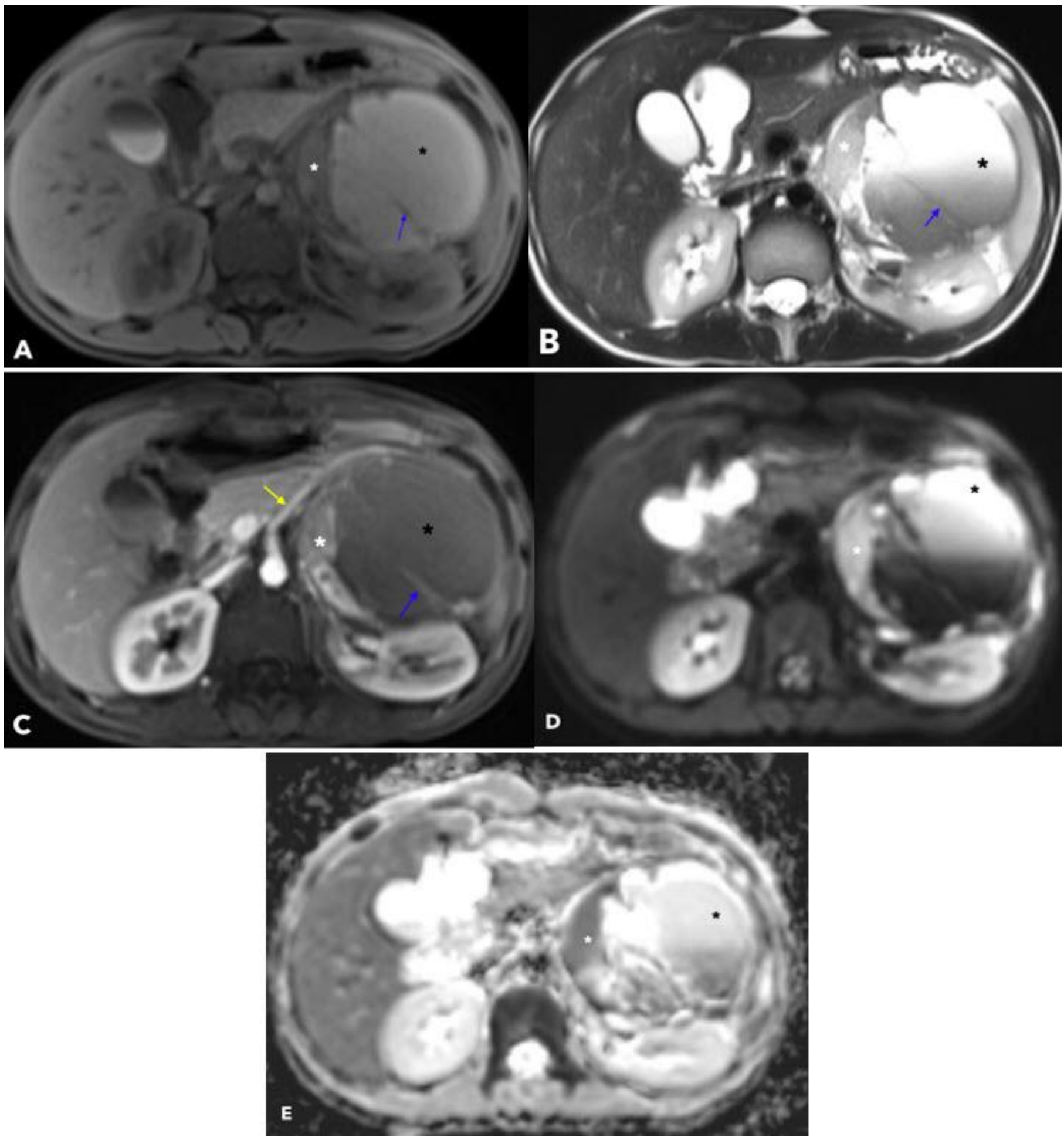

Figure 2: 16 year old female with retroperitoneal alveolar soft part sarcoma.

FINDINGS: the lesion shows heterogeneous hyperintensity on T1w 3D VIBE and T2w (A-B), the solid component (white asterisk), wall and septae (blue arrow) shows enhancement after contrast (C) and the solid component of the lesion shows restricted diffusion in diffusion weighted imaging (D \& E).

TECHNIQUE: Siemens Magnetom Skyra 3T magnet

T1 Vibe fs TR3.3, TE 1.3, (A), T2 HASTE TR 1600, TE 97 (B), DWI TR 5900, TE 52and ADC map (D-E), T1Vibe fs TR3.3, TE 1.3 with contrast (C), Contrast material was Dotarem (gadoterate meglumine) $0.1 \mathrm{mmol} / \mathrm{kg}$ 

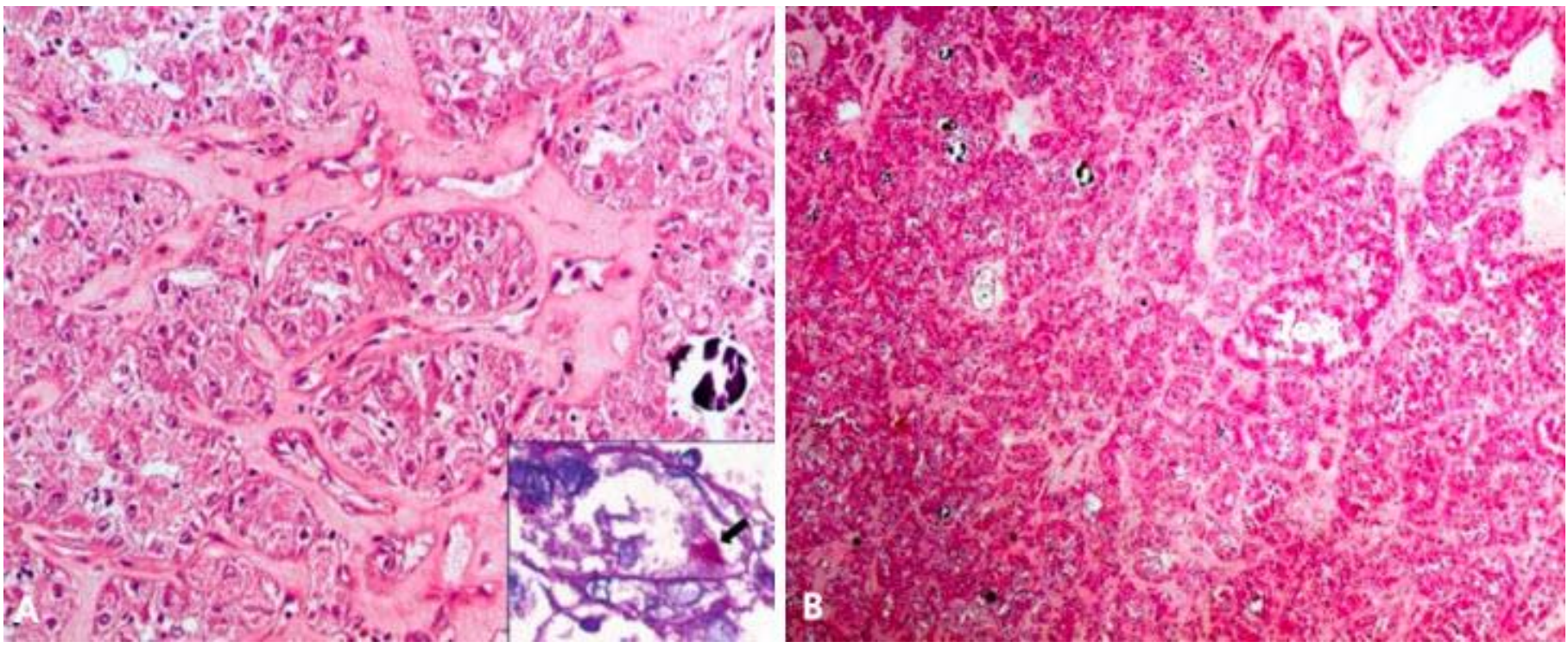

Figure 3: 16 year old female with retroperitoneal alveolar soft part sarcoma.

FINDINGS: Scanner view showing a tumor arranged in an alveolar pattern separated by edematous septae and specks of calcification [H \& E, 40x] (A) Higher magnification shows round to polygonal cells, with abundant granular eosinophilic to vacuolated cytoplasm $(\mathrm{B})$

TECHNIQUE: Microscopic examination using H\&E stain, Medium-power view (40x) and High magnification from the core needle biopsy of retroperitoneal mass.

\begin{tabular}{|c|c|}
\hline Etiology & $\begin{array}{l}\text { Unknown, may be genetic. The two genes involved in alveolar soft part sarcoma (ASPS) are the alveolar soft } \\
\text { part sarcoma critical region1 (ASPSCR1) gene on chromosome 17and Transcription Enhancer Factor } 3 \text { (TEF3) } \\
\text { gene on chromosome X. }\end{array}$ \\
\hline Incidence & $0.5-1 \%$ \\
\hline Gender & Females more than male \\
\hline Age & $15-35$ years, rare in children under 5 or in adults over 50. \\
\hline Risk Factors & No known risk factor \\
\hline Treatment & $\begin{array}{l}\text { Extensive resection of the mass is considered the most effective treatment. Adjunctive radiation treatment and } \\
\text { chemotherapy are used for local recurrences and distant metastases. }\end{array}$ \\
\hline Prognosis & Poor prognosis. \\
\hline $\begin{array}{l}\text { Findings on } \\
\text { imaging }\end{array}$ & $\begin{array}{l}\text { USG: a solid mass with heterogeneous internal echogenicity which shows rapid blood flow on color doppler } \\
\text { ultrasound (CDUS) and exhibits a low resistive index due to arteriovenous shunt within the mass. } \\
\text { CT: a large well defined iso or hypodense mass on the pre contrast scan and shows strong enhancement in post } \\
\text { contrast with central necrosis. The presence of dilated feeding vessels and draining veins around mass is a } \\
\text { pointer towards ASPS. } \\
\text { MRI: a well circumscribed tumor which has heterogeneous signal intensity. The tumor typically has } \\
\text { intermediate signal intensity on T1-weighted images and relatively high signal intensity on T2 weighted } \\
\text { images, with multiple flow voids within. } \\
\text { CDUS, CECT, CEMRI, and angiography all show increased perilesional and intralesional vascularity. } \\
\text { The presence of a large soft-tissue mass associated with large peritumoral vessels is strongly suggestive of } \\
\text { ASPS. } \\
\text { PET-CT: PET-CT is the most helpful imaging modality for detecting primary ASPS. It helps to confirm the } \\
\text { anatomic origin of the ASPS, directs its biopsy, and assess the distribution of disease. Both the primary and } \\
\text { metastatic ASPS show variable but intense FDG uptake on PET-CT. Usually, an SUV max of } 6.8 \text { indicates the } \\
\text { malignant nature of the lesion. }\end{array}$ \\
\hline
\end{tabular}

Table 1: Summary table of alveolar soft part sarcoma of the retroperitoneum. 


\begin{tabular}{|c|c|c|c|}
\hline & USG & CT & MRI \\
\hline $\begin{array}{l}\text { Alveolar soft part } \\
\text { sarcoma of the } \\
\text { retroperitoneum }\end{array}$ & $\begin{array}{l}\text { - Variable echogenicity } \\
\text { - Exhibit rapid blood flow and } \\
\text { low resistive index on color } \\
\text { doppler ultrasonography } \\
\text { (CDUS) }\end{array}$ & $\begin{array}{l}\text { - Well defined } \\
\text { - Iso or hypodense on the pre contrast scan } \\
\text { - Strong enhancement on post contrast } \\
\text { with central necrosis } \\
\text { - The presence of dilated feeding vessel } \\
\text { and draining vein around mass }\end{array}$ & $\begin{array}{l}\text { - A well-defined mass with } \\
\text { Intermediate signal intensity } \\
\text { on T1W and relatively high } \\
\text { signal intensity on T2W. } \\
\text { - Multiple flow voids }\end{array}$ \\
\hline $\begin{array}{l}\text { Renal cell } \\
\text { carcinoma }\end{array}$ & $\begin{array}{l}\text { - Solid or partially cystic mass } \\
\text { - Hyper, iso, or hypoechoic to } \\
\text { the surrounding renal } \\
\text { parenchyma } \\
\text { - Has hypoechoic halo } \\
\text { pseudocapsule }\end{array}$ & $\begin{array}{l}\text { Non contrast computed tomography } \\
\text { (NCCT)- } \\
\text { - Soft tissue attenuation between 20-70 } \\
\text { HU } \\
\text { - Larger lesions have areas of necrosis } \\
\text { - Few show calcification } \\
\text { Contrast enhanced computed tomography } \\
\text { (CECT)- } \\
\text { - Shows enhancement } \\
\text { - Corticomedullary phase of contrast } \\
\text { shows variable enhancement, usually } \\
\text { less than the normal cortex }\end{array}$ & $\begin{array}{l}\text { - T1- Heterogeneous signal } \\
\text { intensity } \\
\text { - T2W- Hyperintense or hypo } \\
\text { intense } \\
\text { - Show contrast enhancement }\end{array}$ \\
\hline $\begin{array}{l}\text { Adrenal cortical } \\
\text { carcinoma }\end{array}$ & $\begin{array}{l}\text { - Well-defined } \\
\text { - Variable appearance based on } \\
\text { the size of the lesion } \\
\text { - Smaller lesions are } \\
\text { homogenous } \\
\text { - Larger lesions are } \\
\text { heterogeneous secondary to } \\
\text { necrosis/hemorrhage. }\end{array}$ & $\begin{array}{l}\text { - Well-defined } \\
\text { - Irregular shaped } \\
\text { - Large mass } \\
\text { - Shows central necrosis, haemorrhage, } \\
\text { or calcification } \\
\text { - Heterogeneous enhancement }\end{array}$ & $\begin{array}{l}\text { - Heterogeneous high signal } \\
\text { intensity on both T1w and } \\
\text { T2w. } \\
\text { - Shows heterogeneous } \\
\text { enhancement on post } \\
\text { contrast } \\
\text { - Areas of hemorrhage and } \\
\text { necrosis may be seen }\end{array}$ \\
\hline $\begin{array}{l}\text { Arteriovenous } \\
\text { malformations }\end{array}$ & - High flow patterns & $\begin{array}{l}\text { - Nidus between arterial and venous } \\
\text { channel without obvious mass } \\
\text { - Abundant vascular channel and scanty } \\
\text { solid component mainly fibrofatty tissue }\end{array}$ & $\begin{array}{l}\text { - Numerous flow voids } \\
\text { - Hyper intense signal without } \\
\text { an obvious mass }\end{array}$ \\
\hline
\end{tabular}

Table 2: Differential diagnosis table for alveolar soft part sarcoma of the retroperitoneum.

\section{ABBREVIATIONS}

ASPS = Alveolar Soft Part Sarcoma

ASPSCR1 = Alveolar Soft Part Sarcoma Critical Region 1

$\mathrm{CT}=$ Computed Tomography

MRI = Magnetic Resonance Imaging

PET-CT $=$ Positron Emission Tomography- Computed

Tomography

TEF3 = Transcription Enhancer Factor 3

\section{KEYWORDS}

Retroperitoneum; Alveolar soft part sarcoma; Adolescent; Pseudoalveolar; Metastasis

\section{Online access}

This publication is online available at: www.radiologycases.com/index.php/radiologycases/article/view/4003

\section{Peer discussion}

Discuss this manuscript in our protected discussion forum at www.radiolopolis.com/forums/JRCR

\section{Interactivity}

This publication is available as an interactive article with scroll, window/level, magnify and more features.

Available online at www.RadiologyCases.com

\section{Published by EduRad}

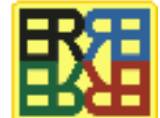

www.EduRad.org 